\title{
Overexpression of CIP2A in clear cell renal cell carcinoma promotes cellular epithelial-mesenchymal transition and is associated with poor prognosis
}

\author{
QIZHEN TANG $^{1}$, QIFEI WANG ${ }^{1}$, GUANG ZENG $^{2}$, QUANLIN LI ${ }^{1}$, TAO JIANG $^{1}$, \\ ZHIWEI ZHANG ${ }^{1}$, WEI ZHENG ${ }^{1}$ and KENAN WANG ${ }^{1}$ \\ ${ }^{1}$ Department of Urology, The First Affiliated Hospital of Dalian Medical University; \\ ${ }^{2}$ Training Office of Graduate School of Dalian Medical University, Dalian, Liaoning 116011, P.R. China
}

Received July 9, 2015; Accepted July 27, 2015

DOI: $10.3892 /$ or.2015.4217

\begin{abstract}
Cancerous inhibitor of protein phosphatase 2A (CIP2A) is a newly characterized oncoprotein involved in a variety of malignant tumors. However, its expression pattern and biological functions in clear cell renal cell carcinoma (ccRCC) remain unclear. In the present study, our findings demonstrated that expressions of CIP2A mRNA and protein in ccRCC tissues and cell lines were significantly higher than those in paired normal renal tissues or normal renal tubular epithelial cells $(\mathrm{P}<0.05)$. High CIP2A level was closely correlated with $T$ stage $(\mathrm{P}=0.001)$, tumor size $(\mathrm{P}=0.009)$, lymph node metastasis $(\mathrm{P}=0.014)$, vascular invasion $(\mathrm{P}=0.018)$ and high Snail expression $(\mathrm{P}<0.001)$. Additionally, ccRCC patients with high CIP2A expression had significantly shorter overall survival (OS, $\mathrm{P}<0.001)$ and disease-free survival (DFS, $\mathrm{P}<0.001$ ) when compared with patients with the low expression of CIP2A. On Cox multivariate analysis, CIP2A overexpression was an independent and significant prognostic factor for OS $(\mathrm{P}=0.010)$ and DFS $(\mathrm{P}=0.004)$. Furthermore, knockdown of the CIP2A expression significantly reduced ccRCC cell invasion, with decreased Snail and Vimentin expression, and increased E-cadherin expression. Taken together, our data identified CIP2A as a critical oncoprotein involved in cell invasion and epithelial mesenchymal transition (EMT), which could serve as a therapeutic target in ccRCC.
\end{abstract}

\section{Introduction}

Renal cell carcinoma (RCC) is the most common carcinoma of the adult kidney, and its incidence has gradually increased

Correspondence to: Dr Qifei Wang, Department of Urology, The First Affiliated Hospital of Dalian Medical University, Dalian, Liaoning 116011, P.R. China

E-mail: wangqifei12345@163.com

Key words: cancerous inhibitor of protein phosphatase 2A, clear cell renal cell carcinoma, epithelial mesenchymal transition, prognosis during the last decades. At least 5 histologic subtypes are encompassed, but majority are cataloged into clear cell RCC (ccRCC), which is responsible for most of the deaths (1). The 5-year survival rate for patients with localized RCC is $\sim 70-90 \%$, but $20-40 \%$ of patients suffer recurrence after surgery dependent on the tumor stage and grade (2). Once metastatic disease develops, the 5-year survival rate decreases to $<20 \%$ (3). The driving factors underlying RCC metastasis remain poorly defined and better understanding of RCC metastasis mechanisms is required for the development of rational strategies for the prevention and treatment of RCC recurrence.

Cancerous inhibitor of protein phosphatase 2A (CIP2A), also known as KIAA1524 and p90, is a recently characterized human oncoprotein which stabilizes c-MYC protein level by inhibiting protein phosphatase $2 \mathrm{~A}$ (PP2A) dephosphorylation activity toward c-MYC serine 62 (S62), thereby restraining c-MYC degradation mediated by PP2A in cancer cells $(4,5)$. Recently it has been associated with poor outcomes in breast, colon, non-small cell lung and hepatocellular cancer (6-10). In renal cell carcinomas, a recent report showed CIP2A promoted tumour invasion and metastasis by regulation of c-MYC expression, and predicted poor survival (11). However, currently, no study has reported CIP2A expression in ccRCC patients and the underlying mechanisms of CIP2A involved in ccRCC progression are need to be further elucidated. Recent studies showed that CIP2A promoted pancreatic ductal adenocarcinoma and cervical-cancer progression by upregulation of epithelial-to-mesenchymal transition (EMT) (11-13). However, whether CIP2A is related with EMT in ccRCC is still unknown.

In the present study, we examined both mRNA and protein expression patterns in ccRCC cell lines and tissues. We also investigated the correlations between CIP2A protein expression and clinicopathologic parameters, and its prognostic value for survival of patients with ccRCC. Moreover, we analyzed the relationship between expression of CIP2A and Snail, a marker of EMT. Then, we employed the small interfering RNA (siRNA) technique to evaluate the effects of knockdown of CIP2A on the invasion and the expression of the EMT marker in a ccRCC cell line in vitro. 


\section{Materials and methods}

Cell lines and clinical sample. ccRCC cell lines (786-O, Caki-1 and Caki-2) and renal tubular epithelial cell line HK2 were obtained from the American Tissue Culture Collection (ATCC) and maintained in recommended media supplemented with $10 \%$ fetal bovine serum (FBS; Hyclone, Logan, UT, USA) and $1 \%$ penicillin/streptomycin (Invitrogen, Carlsbad, CA, USA) at $37^{\circ} \mathrm{C}$ in a humidified atmosphere containing $5 \% \mathrm{CO}_{2}$.

Tumor specimens were collected from two consecutive cohorts of patients with primary ccRCC. Cohort A consisted of 20 patients, from whom fresh tumor samples coupled with adjacent non-tumorous renal tissues $5-10 \mathrm{~cm}$ far from the tumor edge were obtained and subjected to CIP2A mRNA and protein expression analysis. All the fresh specimens were stored at $-80^{\circ} \mathrm{C}$ until use. Cohort B comprised 148 ccRCC patients who underwent curative tumor resection and pathologically proven ccRCC were recruited from the Second Affiliated Hospital of Dalian Medical University between 2003 and 2008. None of the patients had received chemotherapy or radiotherapy prior to surgery. This study was approved by the Institutional Review Board of Dalian Medical University. Written informed consent was obtained from all participants. Clinical stage of these ccRCC patients were classified according to World Health Organization criteria and staged according to the tumor-lymph node-metastasis (TNM) classification system.

All the 148 ccRCC patients received follow-up. The followup period ranged from 72 to 132 months. Seventeen patients was lost of follow-up. The median overall survival (OS) and disease-free survival (DFS) of patients was 65 and 45 months, respectively.

Immunohistochemistry. Tissue sections ( $4 \mu \mathrm{m}$ thick) were obtained from formalin-fixed and paraffin-embedded tissue blocks from the ccRCC samples. Sections were washed in xylene to remove the paraffin, rehydrated with serial dilutions of alcohol, followed by a wash in PBS solution. Endogenous peroxidase activity was blocked by $3 \% \mathrm{H}_{2} \mathrm{O}_{2}$ at $37^{\circ} \mathrm{C}$ for $30 \mathrm{~min}$. Sections were incubated in $10 \%$ normal goat serum to block nonspecific protein binding sites. Sections were then incubated in primary antibodies against CIP2A (1:200) or Snail (1:100) (both from Abcam, Cambridge, MA, USA) overnight at $4^{\circ} \mathrm{C}$. After the primary antibody was washed off, sections were incubated with biotinylated secondary antibodies for $30 \mathrm{~min}$ at $37^{\circ} \mathrm{C}$. Sections were then incubated with streptavidin horseradish peroxidase for $30 \mathrm{~min}$ at $37^{\circ} \mathrm{C}$. 3,3-Diaminobenzidine (DAB) substrate was applied to the section, and then they were counterstained with hematoxylin. Negative controls of immunohistochemical reactions included omission of the primary antibody.

Immunohistochemistry evaluation and selection of the cut-off score. The immunostaining was examined under a light microscope by two pathologists blinded to the experimental conditions. Each section was assigned an intensity score from 0-3 (0 for no staining, 1 for weak staining, 2 for moderate staining, and 3 for strong staining) and proportion of tumor cells for that intensity over the total number of tumor cells was recorded as $5 \%$ increments from a range of $0-100$. A final score (range 0-300) was achieved by adding the sum of scores obtained for each intensity and proportion of area stained. ROC curve analysis was used to determine the cut-off value for CIP2A or Snail expression in the training set using the 0, 1-criterion. In the CIP2A or Snail score, the sensitivity and specificity for survival status under the study was plotted to generate the ROC curve (Fig. 1). The score (145 for CIP2A and 125 for Snail) closest to the points of maximum sensitivity and maximum specificity was selected as the cut-off value. The tumors designated as low expression for CIP2A or Snail were those with scores below the cut-off value, high expression was the scores above or equal to the value.

RNA preparation and quantitative RT-PCR ( $q R T-P C R)$. Total RNA was extracted using the TRIzol reagent (Invitrogen) according to the manufacturer's protocol. Primers for CIP2A were as follows: forward, 5'-GGGAATTCCCTGATTCCTCT TCA-3' and reverse, 5'-CCCTCGAGCTAGAAGCTTACTTC CAT-3'; for $\beta$-actin forward, 5'-GCCAACACAGTGCTGTC TGG-3' and reverse, 5'-GCTCAGGAGGAGCAATGATC TTG-3'. Reverse transcription PCR was done using the PrimeScript RT reagent kit (Takara, Dalian, China). qRT-PCR was performed using SYBR Premix Ex Taq II (Takara) and the ABI Prism ${ }^{\circledR} 7900$ sequence detection system (Applied Biosystems, Foster City, CA, USA). $\beta$-actin levels were used as internal controls, and fold-changes were calculated using the $2^{-\Delta \Delta \mathrm{Ct}}$ method. Each experiment was performed in triplicate.

Western blot analysis. Cells were washed with PBS and lysed in RIPA buffer (Sigma Chemical Co., St. Louis, MO, USA) and centrifuged for $15 \mathrm{~min}$. At $4^{\circ} \mathrm{C}$ the supernatant was transferred to a fresh tube. For western blot analysis, equal amounts of total protein were mixed with $2 X$ SDS sample buffer, incubated at $100^{\circ} \mathrm{C}$ for $5 \mathrm{~min}$ and separated by SDS-PAGE. After electrophoresis, protein was blotted onto a PVDF membrane (Millipore Co., Billerica, MA, USA) and blocked for $1 \mathrm{~h}$ at room temperature and was then incubated with antibodies against CIP2A (1:2,000 dilution), Snail (1:1,000 dilution), E-cadherin (1:1,000 dilution), vimentin (1:1,000 dilution) and $\beta$-actin (1:1,000 dilution) (all from Abcam) with a dilution of 1:1,000 at $4^{\circ} \mathrm{C}$ overnight. The blots were incubated with the horseradish peroxidase-conjugated secondary antibody (Sigma Chemical Co.) with a dilution of 1:1,000 after repeated washing with a blocking buffer. The antigen-antibody complexes were detected by using an ECL kit (Abcam) according to the manufacturer's recommendations. All experiments were performed as triplicates.

Oligonucleotide transfection. The siRNA targeting CIP2A and control siRNA were purchased from Invitrogen. The siRNA sequence for CIP2A was 5'-GACAACUGUCAAGUGU ACCACUCUU-3'. Cells were transfected with either CIP2A or control siRNA using Oligofectamine reagent (Invitrogen) according to the manufacturer's instructions. Following transfection, the mRNA and protein levels were assessed $48 \mathrm{~h}$ later.

Cell invasion assays. Cell invasion assays were performed by using Transwell chambers. Cells $\left(1 \times 10^{5}\right)$ were added into the upper chamber of 8 micron Transwells precoated with Matrigel (BD Bioscience, San Jose, CA, USA). Cells were plated in 
A

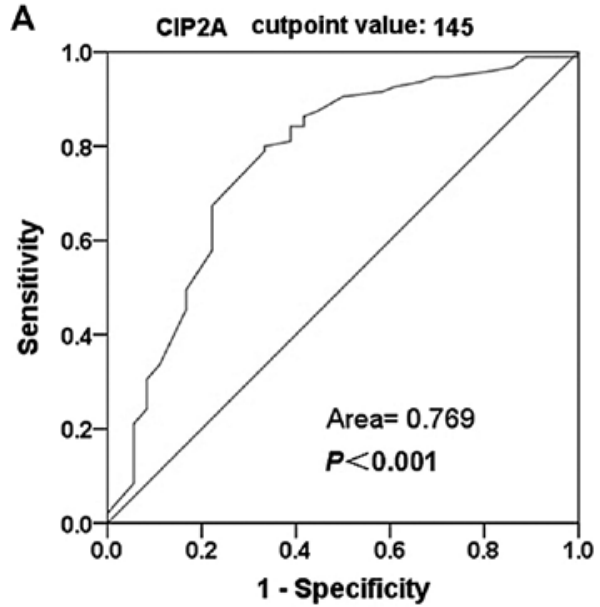

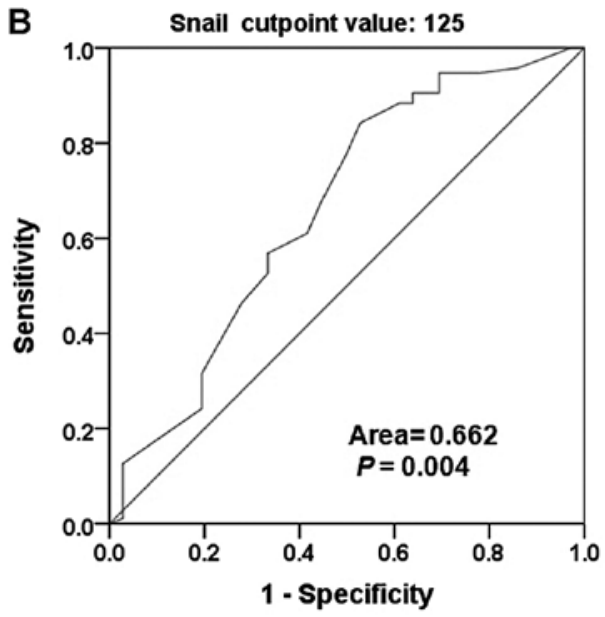

Figure 1. Receiver operating characteristic curves were created to determine the cut-off score for positive expression of (A) cancerous inhibitor of protein phosphatase 2A (CIP2A) and (B) Snail in clear cell renal cell carcinoma (ccRCC). The sensitivity and specificity for survival status was plotted and the areas under curve (AUCs) were indicated.

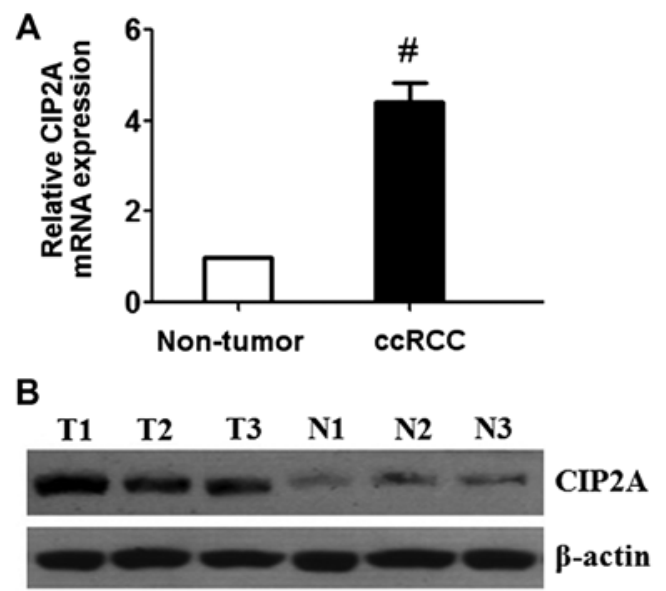

Figure 2. Analysis of cancerous inhibitor of protein phosphatase $2 \mathrm{~A}$ (CIP2A) mRNA and protein expression in 20 cases of clear cell renal cell carcinoma (ccRCC) tissues and adjacent normal renal tissues. (A) qRT-PCR detection of CIP2A mRNA expression. (B) Western blot detection of CIP2A protein expression. $\beta$-actin was used as an internal control. Each assay was performed at least three times. Data are presented as mean \pm SD. Differences were assessed by one way ANOVA. ${ }^{\text {"P }} \mathrm{P}<0.05$ was considered to indicate a statistically significant.

medium without serum, and medium containing 10\% FBS in the lower chamber served as chemoattractant. After $24 \mathrm{~h}$ incubation, the cells that did not invade through the pores were carefully wiped off. Then the filters were fixed in $90 \%$ alcohol, followed by crystal violet stain. Ten random fields were counted per chamber by using an inverted microscope (Olympus, Tokyo, Japan), and each experiment was repeated three times.

Statistical analysis. The significance of the relationships between CIP2A protein expression and clinicopathological parameters and Snail protein expression were evaluated using the Chi-square test. DFS and OS curves were calculated using the Kaplan-Meier method and compared by log-rank test. Multivariate analysis was used to identify independent prognostic factors for DFS and OS by using the Cox proportional hazards regression model. All the other data from three independent experiments were expressed as mean \pm SD and were analyzed by one way ANOVA. SPSS 16.0 software (SPSS, Inc., Chicago, IL, USA) was used for statistical analysis. A value of $\mathrm{P}<0.05$ was considered statistically significant.

\section{Results}

Expression of CIP2A $\mathrm{mRNA}$ and protein in ccRCC tissues. We first examined CIP2A mRNA expression in $20 \mathrm{ccRCC}$ tissues and paired adjacent normal renal tissues by qRT-PCR. The relative level of CIP2A mRNA in ccRCC tissues was significantly higher than that in adjacent normal renal tissues $(\mathrm{P}<0.05)$ (Fig. 2A). To investigate whether CIP2A was also elevated at the protein level, western blot analysis was performed on the tissues. We found that the relative level of CIP2A protein in ccRCC tissues was significantly higher than that in adjacent normal renal tissues $(\mathrm{P}<0.05)$ (Fig. 2B), consistent with the results of qRT-PCR.

Immunohistochemical analysis of CIP $2 A$ expression in ccRCC samples and its relationship to clinicopathological parameters. We further analyzed CIP2A protein level in 148 ccRCC tissues using an immunohistochemical approach. The CIP2A protein appeared to be mainly expressed in cytoplasmic components of tumor cells (Fig. 3). Of these tumor tissues, 97 (65.5\%) of the cases showed CIP2A-high expression. The relationship between CIP2A protein expression and various clinicopathological parameters is described in Table I. CIP2A protein expression significantly correlated with $\mathrm{T}$ stage $(\mathrm{P}=0.001)$, tumor size $(\mathrm{P}=0.009)$, lymph node metastasis $(\mathrm{P}=0.014)$ and vascular invasion $(\mathrm{P}=0.018)$. However, CIP2A protein expression was not associated with other clinicopathological features such as age, gender and tobacco smoking.

Association between the protein expression of CIP $2 A$ and Snail in ccRCC tissues. We analyzed Snail protein level in 148 ccRCC tissues by immunohistochemistry. The Snail protein appeared to be mainly expressed in cytoplasmic and nuclear components of tumor cells (Fig. 3). Of these tumor 
Table I. Association of CIP2A expression with clinicopathological features of ccRCC.

\begin{tabular}{|c|c|c|c|c|}
\hline \multirow[b]{2}{*}{ Features } & \multirow[b]{2}{*}{ No. of cases } & \multicolumn{2}{|c|}{ CIP2A } & \multirow[b]{2}{*}{ P-value ${ }^{a}$} \\
\hline & & High expression, $\mathrm{n}(\%)$ & Low expression, $\mathrm{n}(\%)$ & \\
\hline Age at diagnosis & & & & 0.365 \\
\hline$<55$ & 65 & $40(61.5)$ & $25(38.5)$ & \\
\hline$\geq 55$ & 83 & $57(68.7)$ & $26(31.3)$ & \\
\hline Gender & & & & 0.311 \\
\hline Female & 50 & $30(60.0)$ & $20(40.0)$ & \\
\hline Male & 98 & $67(68.4)$ & $31(31.6)$ & \\
\hline Tobacco smoking & & & & 0.235 \\
\hline Absent & 101 & $63(62.4)$ & $38(37.6)$ & \\
\hline Present & 47 & $34(72.3)$ & $13(27.7)$ & \\
\hline T stage & & & & 0.001 \\
\hline $\mathrm{T}_{1,2}$ & 94 & $52(55.3)$ & $42(44.7)$ & \\
\hline $\mathrm{T}_{3,4}$ & 54 & $45(83.3)$ & $9(16.7)$ & \\
\hline Tumor size (cm) & & & & 0.009 \\
\hline$<4$ & 71 & $39(54.9)$ & $32(45.1)$ & \\
\hline$\geq 4$ & 77 & $58(75.3)$ & $19(24.7)$ & \\
\hline Lymph node metastasis & & & & 0.014 \\
\hline Absent & 103 & $61(59.2)$ & $42(40.8)$ & \\
\hline Present & 45 & $36(80.0)$ & $9(20.0)$ & \\
\hline Vascular invasion & & & & 0.018 \\
\hline Absent & 107 & $64(59.8)$ & $43(40.2)$ & \\
\hline Present & 41 & $33(80.5)$ & $8(19.5)$ & \\
\hline
\end{tabular}

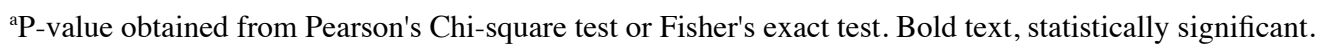

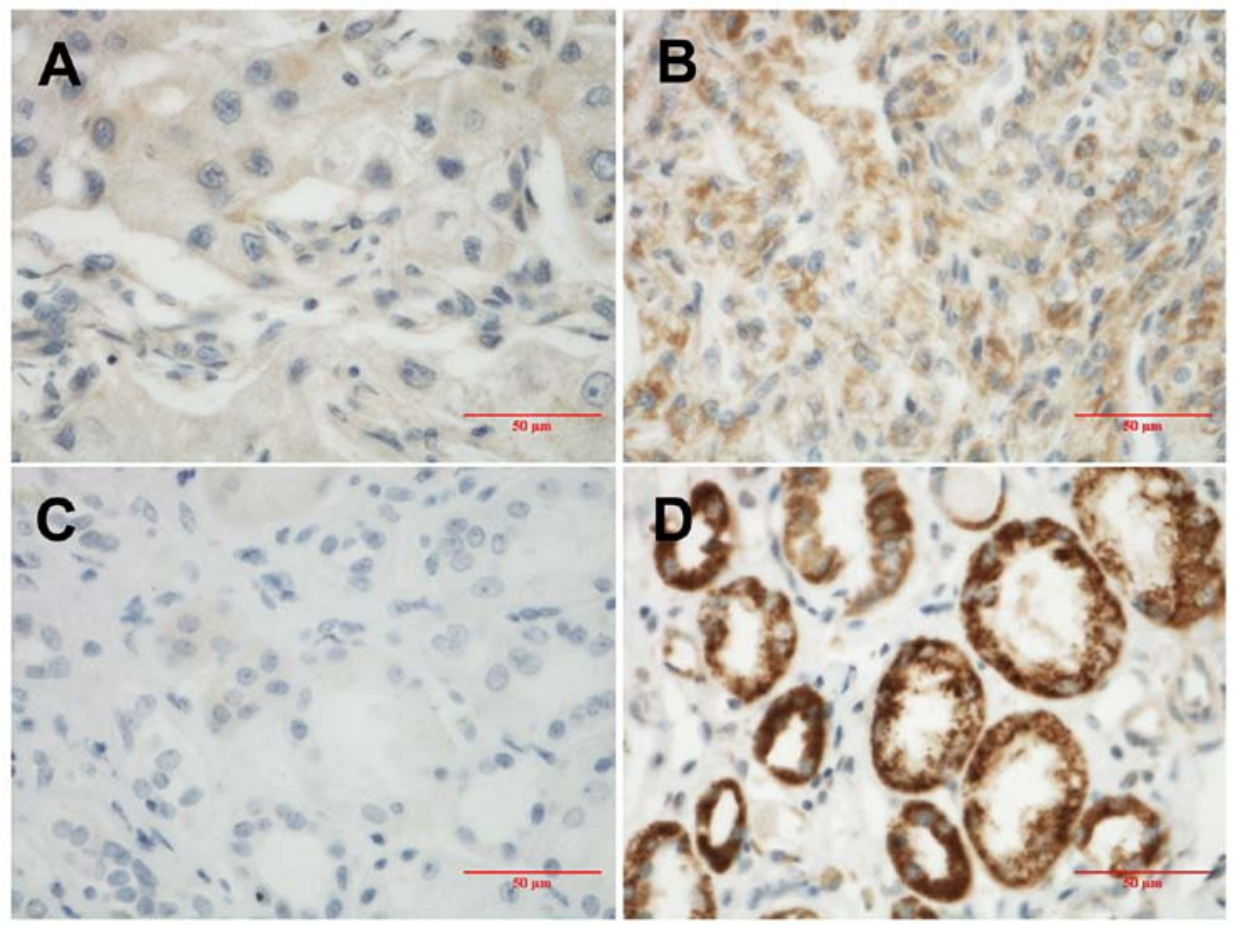

Figure 3. Immunohistochemical analysis of cancerous inhibitor of protein phosphatase 2A (CIP2A) and Snail expression in clear cell renal cell carcinoma (ccRCC) patients. (A) Low expression level of CIP2A. (B) High expression level of CIP2A. (C) Low expression level of Snail. (D) High expression level of Snail. Original magnification, $\mathrm{x} 400$. 
A

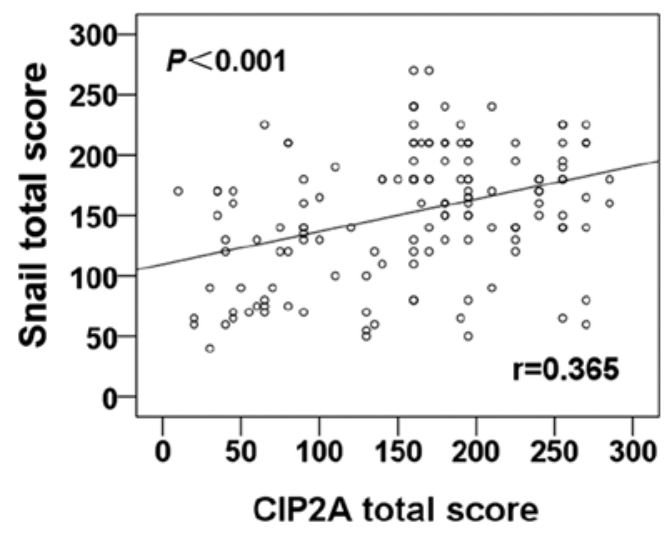

B

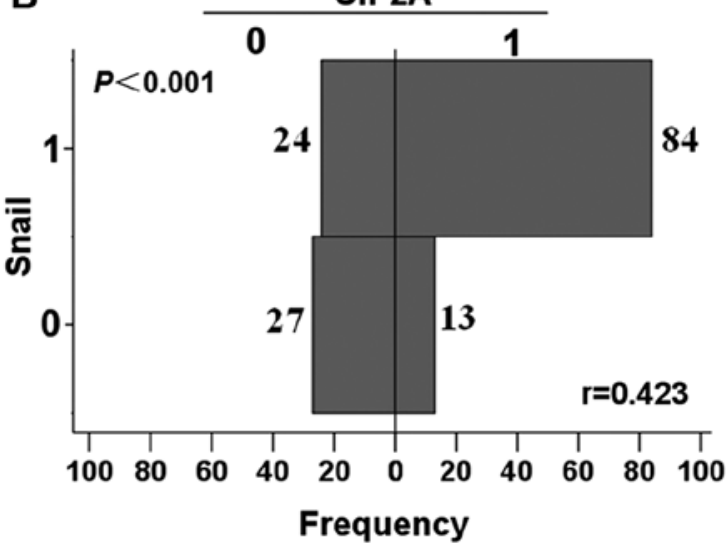

Figure 4. The association between the protein expression of cancerous inhibitor of protein phosphatase 2A (CIP2A) and Snail in clear cell renal cell carcinoma (ccRCC). (A) Pearson correlation analysis estimates of the correlation between CIP2A and Snail expression. $\mathrm{r}=0.365$, P<0.001. (B) Snail (high) and Snail (low) expression in ccRCC with CIP2A (high) and CIP2A (low) expression. $\chi^{2}=26.496, \mathrm{P}<0.001$.
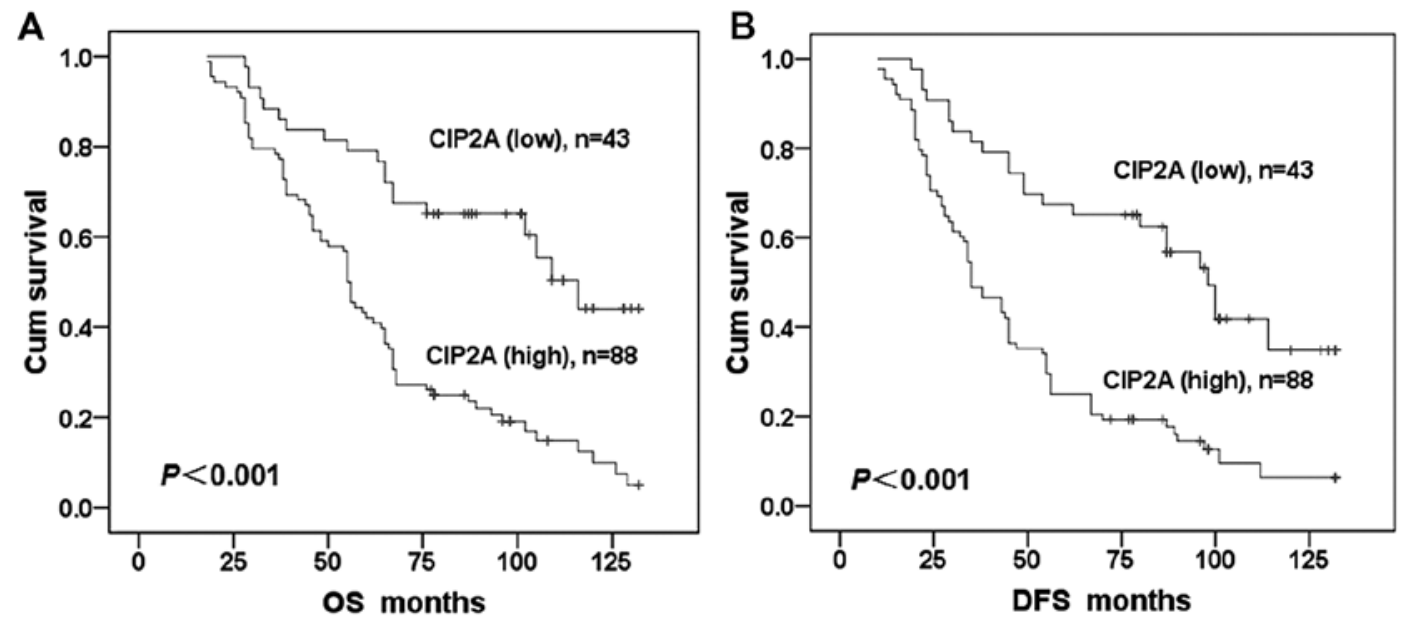

Figure 5. The overall survival (OS) (A) and disease-free survival (DFS) (B) rate of patients with clear cell renal cell carcinoma (ccRCC) estimated according to the cancerous inhibitor of protein phosphatase 2A (CIP2A) expression level in ccRCC samples (Kaplan-Meier method) with immunohistochemical staining. The patients with high CIP2A expression tended to have a significantly shorter OS and DFS than those with low CIP2A expression.

tissues, $108(72.9 \%)$ cases showed Snail-high expression. Pearson correlation analysis was used to analyze the association between the total score of CIP2A and Snail in ccRCC. As shown in Fig. 4, there was significant correlation between the total score of CIP2A and Snail in ccRCC tissues $(r=0.365$, $\mathrm{P}<0.001$ ) (Fig. 4A). Furthermore, in ccRCC with high Snail expression, high expression of CIP2A was observed in $84(77.8 \%)$ of 108 cases, and in ccRCC with low snail expression, high expression of CIP2A was observed in 13 (32.5\%) of 40 cases. Chi-squared tests showed CIP2A high expression occurred significantly more frequently in high Snail expression ccRCC tissues than in the low Snail expression ccRCC tissues $\left(\chi^{2}=26.496, \mathrm{P}<0.001\right)$ (Fig. 4B).

CIP2A expression and patient survival. The prognostic value of CIP2A for OS and DFS in ccRCC patients were evaluated by comparing the patients with high and low CIP2A expression. According to the Kaplan-Meier survival analysis, ccRCC patients with high CIP2A expression had obviously lower OS and DFS rates than did those with CIP2A low expression (Fig. 5).
Upon univariate analysis with the Cox proportional hazards model, gender $(\mathrm{P}=0.046)$, tumor size $(\mathrm{P}=0.005)$, tobacco smoking $(\mathrm{P}=0.023)$, $\mathrm{T}$ stage $(\mathrm{P}<0.001)$, lymph node metastasis $(\mathrm{P}<0.001)$, vascular invasion $(\mathrm{P}<0.001)$, Snail expression $(\mathrm{P}=0.001)$ and CIP2A expression $(\mathrm{P}<0.001)$ were all positively correlated with a shorter OS and tumor size $(\mathrm{P}=0.028)$, tobacco smoking $(\mathrm{P}=0.022)$, $\mathrm{T}$ stage $(\mathrm{P}<0.001)$, lymph node metastasis $(\mathrm{P}<0.001)$, vascular invasion $(\mathrm{P}<0.001)$, Snail expression $(\mathrm{P}=0.002)$ and $\mathrm{CIP} 2 \mathrm{~A}$ expression $(\mathrm{P}<0.001)$ were all positively correlated with a shorter DFS. Multivariate analyses revealed that $\mathrm{T}$ stage $(\mathrm{P}=0.027)$, lymph node metastasis $(\mathrm{P}=0.028)$ and CIP2A expression $(\mathrm{P}=0.017)$ were independent prognostic factors for OS and tobacco smoking $(\mathrm{P}=0.049)$, lymph node metastasis $(\mathrm{P}=0.036)$ and CIP2A expression $(\mathrm{P}=0.007)$ for DFS (Table II). Thus, CIP2A expression may be useful for predicting the overall survival of ccRCC patients.

CIP2A expression in ccRCC cell lines. We also used qRT-PCR and western blot analysis to detect the expression of CIP2A mRNA and protein in four ccRCC cell lines as well as in an 
Table II. Cox regression analysis of clinicopathological data correlated with OS and DFS in ccRCC.

\begin{tabular}{|c|c|c|c|c|c|c|c|c|}
\hline \multirow[b]{3}{*}{ Factor } & \multicolumn{4}{|c|}{ OS } & \multicolumn{4}{|c|}{ DFS } \\
\hline & \multicolumn{2}{|c|}{ Univariate } & \multicolumn{2}{|c|}{ Multivariate } & \multicolumn{2}{|c|}{ Univariate } & \multicolumn{2}{|c|}{ Multivariate } \\
\hline & $\mathrm{RR}$ & P-value & $\mathrm{RR}$ & $\mathrm{P}$-value ${ }^{\mathrm{b}}$ & $\mathrm{RR}$ & P-value ${ }^{a}$ & RR & $\mathrm{P}$-value ${ }^{\mathrm{b}}$ \\
\hline Age, years $(\geq 55 /<55)$ & 1.231 & 0.321 & 0.982 & 0.934 & 1.235 & 0.298 & 0.908 & 0.975 \\
\hline Gender (male/female) & 1.578 & 0.046 & 1.282 & 0.307 & 1.418 & 0.112 & - & - \\
\hline Tumor size, $\mathrm{cm}(\geq 4 /<4)$ & 1.805 & 0.005 & 1.098 & 0.682 & 1.562 & 0.028 & 0.975 & 0.993 \\
\hline T stage $\left(\mathrm{T}_{3,4} / \mathrm{T}_{1,2}\right)$ & 3.257 & $<0.001$ & 1.767 & 0.027 & 2.744 & $<0.001$ & 1.558 & 0.076 \\
\hline Tobacco smoking (present/absent) & 1.615 & $\mathbf{0 . 0 2 3}$ & 1.498 & 0.085 & 1.615 & 0.022 & 1.548 & 0.049 \\
\hline Lymph node metastasis (present/absent) & 4.848 & $<0.001$ & 2.580 & 0.008 & 4.002 & $<0.001$ & 2.124 & 0.036 \\
\hline Vascular invasion (present/absent) & 4.332 & $<0.001$ & 1.370 & 0.352 & 3.860 & $<0.001$ & 1.454 & 0.275 \\
\hline CIP2A (high/low) & 3.162 & $<0.001$ & 1.975 & 0.017 & 3.007 & $<0.001$ & 2.064 & 0.007 \\
\hline Snail (high/low) & 2.515 & 0.001 & 1.124 & 0.717 & 2.149 & 0.002 & 1.072 & 0.814 \\
\hline
\end{tabular}

${ }^{a} \mathrm{P}$-value and RR were assessed using univariate Cox regression analysis; ${ }^{\mathrm{b}} \mathrm{P}$-value and RR were assessed using multivariate Cox regression analysis. RR, relative risk. Bold text, statistically significant.

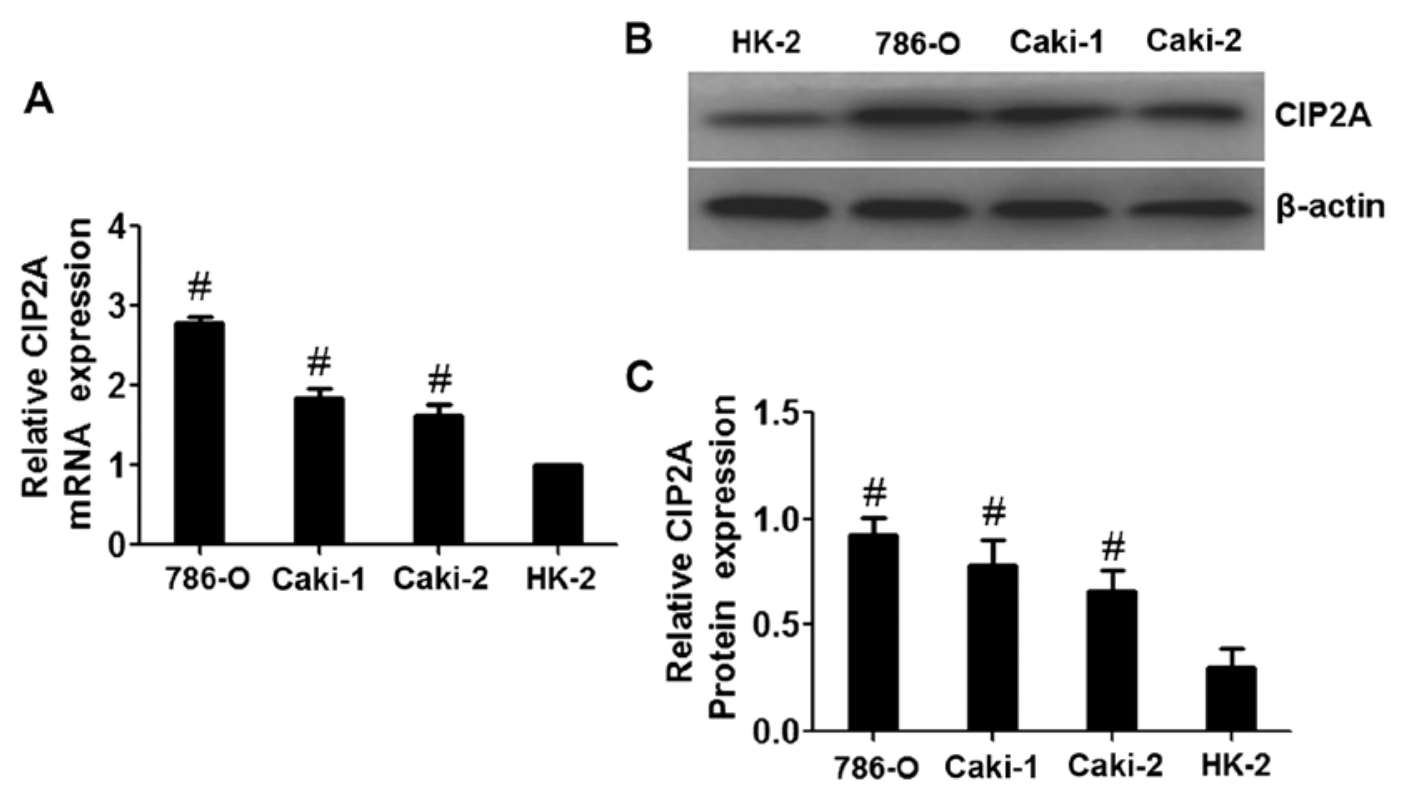

Figure 6. Expression of cancerous inhibitor of protein phosphatase 2A (CIP2A) mRNA and protein in clear cell renal cell carcinoma (ccRCC) cell lines (A) CIP2A mRNA and (B,C) protein expression detected in several ccRCC cell lines (786-O, Caki-1 and Caki-2) as well as in a renal tubular epithelial cell line HK-2 by RT-PCR and western blot analysis. Each experiment was repeated three times. Data are presented as mean \pm SD. Differences were assessed by one way ANOVA. ${ }^{*} \mathrm{P}<0.05$ was considered statistically significant.

renal tubular epithelial cell line HK-2. The 786-O, Caki-1 and Caki-2 showed higher level of CIP2A mRNA relative to renal tubular epithelial cell line HK-2 likewise, CIP2A protein expression was elevated in these ccRCC cell lines compared to the HK-2 cell line (Fig. 6).

Effects of CIP2A depletion on the expression of EMT markers and invasion in vitro. To explore the biological significance of CIP2A in ccRCC, we specifically knocked down its expression in 786-O cells using RNA interference (RNAi). This ccRCC cell line was chosen because of its high abundance of CIP2A. The efficacy of CIP2A siRNA for knockdown of
CIP2A mRNA and protein was confirmed by RT-PCR and western blot analysis, respectively. We observed that CIP2A mRNA and protein levels were significantly reduced in cells transfected with specific siRNA for CIP2A compared with those transfected with control siRNA (Fig. 7A and B). Thus, the CIP2A siRNA could effectively knock down CIP2A expression at both transcriptional and translational levels. Furthermore, knockdown of CIP2A by siRNA resulted in inhibition of Snail and vimentin protein expression, while promoting the E-cadherin expression (Fig. 7C). In addition, Matrigel invasion assay showed that downregulation of CIP2A suppressed the invasiveness of 786-O cells (Fig. 7D). The 

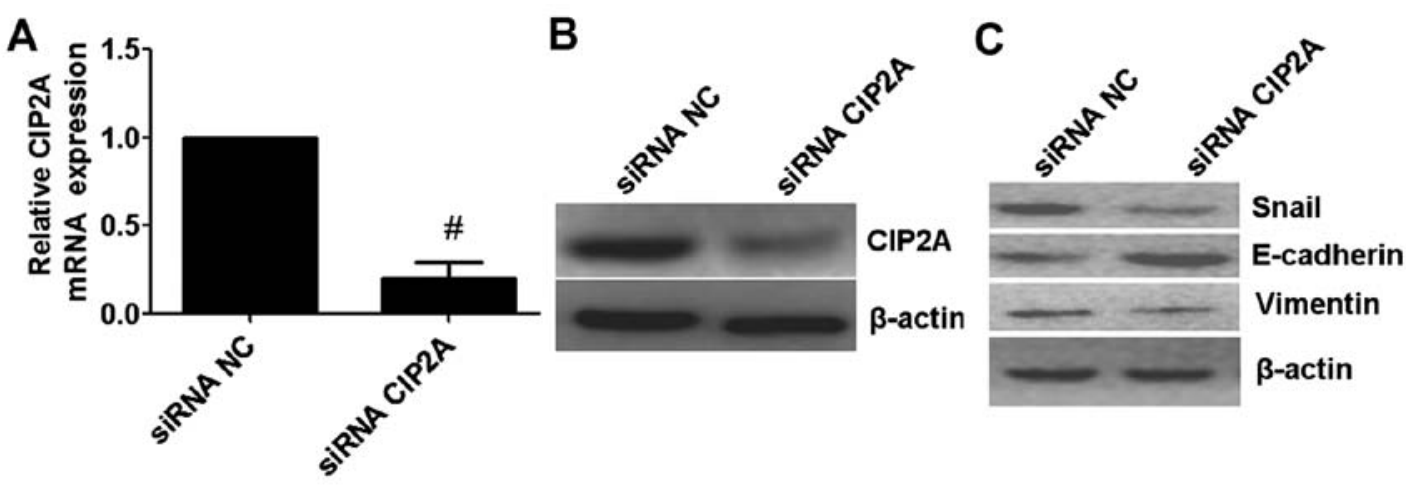

D
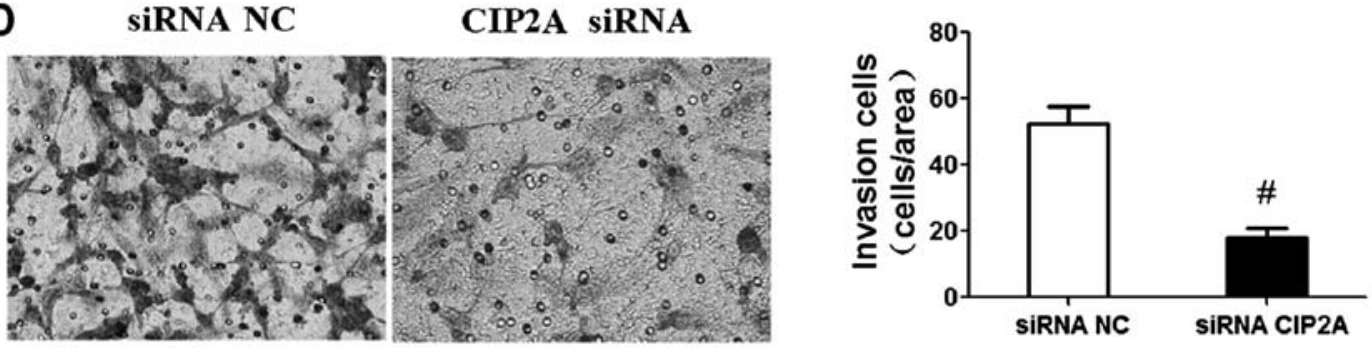

Figure 7. (A) Effects of cancerous inhibitor of protein phosphatase $2 \mathrm{~A}$ (CIP2A) depletion on cell invasion in vitro. (B and C) qRT-PCR analysis of CIP2A mRNA expression in 786-O cells transfected with the specific siRNA targeting CIP2A for $48 \mathrm{~h}$. Western blot analysis of CIP2A and EMT marker expression in 786-O cells transfected with the CIP2A siRNA for $72 \mathrm{~h}$. (D) Matrigel invasion assays. Representative images are shown on the left, and the quantification of ten randomly selected fields is shown on the right. Each experiment was repeated three times. Data are presented as mean \pm SD. Differences were assessed by one way ANOVA. ${ }^{~} \mathrm{P}<0.05$ was considered statistically significant.

average cell counts crossing Matrigel-coated membrane in one high-power field were $52.34 \pm 5.34$ for the control siRNA group and $18.23 \pm 2.45$ for the CIP2A siRNA group $(\mathrm{P}<0.05)$.

\section{Discussion}

In this study, we found CIP2A was upregulated in both ccRCC cell lines and clinical samples, and those ccRCC patients with high CIP2A expression exhibited the poorer survival rates. CIP2A expression was positively related with snail expression, a marker of EMT in ccRCC tissues. In vitro experiments, silencing CIP2A inhibited invasion in the ccRCC cell line, with downregulated expression of mesenchymal marker, and upregulated the expression of the epithelial marker.

In agreement with multiple previous studies $(7,8,10)$. We confirmed that high CIP2A expression is positively correlated with high $\mathrm{T}$ stage, larger tumors, lymph node metastasis, vascular invasion and poor survival, which implies that CIP2A plays an important role in ccRCC progression and serves as an independent biomarker for a poor clinical prognosis.

The main underlying mechanism of CIP2A involved in cancer progression are to inhibit PP2A activity toward the oncogenic transcription factor c-MYC Ser62 and thereby stabilizes the c-MYC protein by preventing its proteolytic degradation $(4,5)$. However, the exact mechanism need to be further investigated. EMT is the process by which polarized epithelial cells are converted into motile mesenchymal cells, with the alterations in adhesion, morphology, cellular architecture and migration potential during this process (14). In recent years, this process has also been shown to apply to the progression and metastasis of a wide variety of malignant tumors, including RCC (15-19) and targeting EMT processes is therefore a promising strategy to block the transition to metastatic phenotype and improve the outcome of the patients. The recent reports showed that CIP2A expression correlates with EMT in various cancers $(12,13)$. However, it is still unclear whether expression of CIP2A correlates with the EMT in ccRCC. In present study, we found that CIP2A expression was significantly related with the expression of Snail, a key EMT marker in ccRCC tissues. Furthermore, in vitro, silencing CIP2A inhibited invasion in the ccRCC cell line, with downregulated expression of mesenchymal markers, including vimentin and Snail, while upregulated expression of epithelial markers, such as E-cadherin. These results indicate CIP2A also correlates with the EMT in ccRCC.

In conclusion, our results indicate CIP2A was overexpressed in ccRCC tissues and this overexpression positively correlates with the aggressive phenotype and predicts poor survival outcome in ccRCC patients. Our results also showed that high CIP2A expression correlates with altered expression of EMT markers in ccRCC. Moreover, we have also presented experimental evidence that depletion of CIP2A in human ccRCC cell line 786-O inhibited cell invasion and the expression of mesenchymal markers, while upregulated expression of epithelial markers. On the basis of the data presented here, CIP2A may serve as a molecular target for future development of ccRCC therapeutics.

\section{Acknowledgements}

This study was supported by the National Science Foundation, grant nos. 21272032 and 30672091. 


\section{References}

1. Lohse CM and Cheville JC: A review of prognostic pathologic features and algorithms for patients treated surgically for renal cell carcinoma. Clin Lab Med 25: 433-464, 2005.

2. Stewart GD, O'Mahony FC, Powles T, Riddick AC, Harrison DJ and Faratian D: What can molecular pathology contribute to the management of renal cell carcinoma? Nat Rev Urol 8: 255-265, 2011.

3. Xu L, Zhu Y, Chen L, An H, Zhang W, Wang G, Lin Z and Xu J: Prognostic value of diametrically polarized tumor-associated macrophages in renal cell carcinoma. Ann Surg Oncol 21: 3142-3150, 2014

4. Junttila MR, Puustinen $P$, Niemelä $M$, Ahola R, Arnold $H$, Böttzauw T, Ala-aho R, Nielsen C, Ivaska J, Taya Y, et al: CIP2A inhibits PP2A in human malignancies. Cell 130: 51-62, 2007.

5. Junttila MR and Westermarck J: Mechanisms of MYC stabilization in human malignancies. Cell Cycle 7: 592-596, 2008.

6. Côme C, Laine A, Chanrion M, Edgren H, Mattila E, Liu X, Jonkers J, Ivaska J, Isola J, Darbon JM, et al: CIP2A is associated with human breast cancer aggressivity. Clin Cancer Res 15 5092-5100, 2009.

7. Dong QZ, Wang Y, Dong XJ, Li ZX, Tang ZP, Cui QZ and Wang EH: CIP2A is overexpressed in non-small cell lung cancer and correlates with poor prognosis. Ann Surg Oncol 18: 857-865, 2011.

8. He H, Wu G, Li W, Cao Y and Liu Y: CIP2A is highly expressed in hepatocellular carcinoma and predicts poor prognosis. Diagn Mol Pathol 21: 143-149, 2012.

9. Niemelä M, Kauko O, Sihto H, Mpindi JP, Nicorici D, Pernilä P, Kallioniemi OP, Joensuu H, Hautaniemi S and Westermarck J: CIP2A signature reveals the MYC dependency of CIP2Aregulated phenotypes and its clinical association with breast cancer subtypes. Oncogene 31: 4266-4278, 2012.

10. Teng HW, Yang SH, Lin JK, Chen WS, Lin TC, Jiang JK, Yen CC, Li AF, Chen PC, Lan YT, et al: CIP2A is a predictor of poor prognosis in colon cancer. J Gastrointest Surg 16: 1037-1047, 2012 .
11. Ren J, Li W, Yan L, Jiao W, Tian S, Li D, Tang Y, Gu G, Liu H and $\mathrm{Xu} \mathrm{Z}$ : Expression of CIP2A in renal cell carcinomas correlates with tumour invasion, metastasis and patients' survival. Br J Cancer 105: 1905-1911, 2011.

12. Wang L, Gu F, Ma N, Zhang L, Bian JM and Cao HY: CIP2A expression is associated with altered expression of epithelialmesenchymal transition markers and predictive of poor prognosis in pancreatic ductal adenocarcinoma. Tumour Biol 34: 2309-2313, 2013.

13. Wu Y, Gu TT and Zheng PS: CIP2A cooperates with H-Ras to promote epithelial-mesenchymal transition in cervical-cancer progression. Cancer Lett 356 (2 Pt B): 646-655, 2015.

14. Kalluri R: EMT: When epithelial cells decide to become mesenchymal-like cells. J Clin Invest 119: 1417-1419, 2009.

15. Kosaka T, Kikuchi E, Mikami S, Miyajima A, Shirotake S, Ishida M, Okada Y and Oya M: Expression of snail in upper urinary tract urothelial carcinoma: Prognostic significance and implications for tumor invasion. Clin Cancer Res 16: 5814-5823, 2010.

16. Li Y, Wang W, Wang W, Yang R, Wang T, Su T, Weng D, Taos T, $\mathrm{Li} \mathrm{W}, \mathrm{Ma} \mathrm{D}$, et al: Correlation of TWIST2 up-regulation and epithelial-mesenchymal transition during tumorigenesis and progression of cervical carcinoma. Gynecol Oncol 124: 112-118, 2012.

17. Mikami S, Katsube K, Oya M, Ishida M, Kosaka T, Mizuno R, Mukai M and Okada Y: Expression of Snail and Slug in renal cell carcinoma: E-cadherin repressor Snail is associated with cancer invasion and prognosis. Lab Invest 91: 1443-1458, 2011.

18. Pantuck AJ, An J, Liu H and Rettig MB: NF-kappaB-dependent plasticity of the epithelial to mesenchymal transition induced by Von Hippel-Lindau inactivation in renal cell carcinomas. Cancer Res 70: 752-761, 2010.

19. Tun HW, Marlow LA, von Roemeling CA, Cooper SJ, Kreinest P, Wu K, Luxon BA, Sinha M, Anastasiadis PZ and Copland JA: Pathway signature and cellular differentiation in clear cell renal cell carcinoma. PLoS One 5: e10696, 2010. 